\title{
Role of NLRP3 Inflammasomes in Neuroinflammation Diseases
}

\author{
Sen Lin Xifan Mei \\ Department of Orthopedic, First Affiliated Hospital of Jinzhou Medical University, Jinzhou, PR China
}

\section{Keywords}

NLRP3 inflammasomes · Spinal cord injury .

Neuroinflammation diseases

\begin{abstract}
Background: Inflammasomes are large intracellular multiprotein signaling complexes that are formed in the cytosolic compartment as an inflammatory immune response to endogenous danger signals. The formation of the inflammasome enables activation of an inflammatory protease caspase- 1 and pyroptosis initiation with the subsequent cleaving of the pro-inflammatory cytokines interleukin (IL)- $1 \beta$ and prolL-18 to produce active forms. The inflammasome complex consists of a nod-like receptor, the adapter apoptosisassociated speck-like protein, and caspase-1. Dysregulation of NLRP3 inflammasome activation is involved in neuroinflammation disease pathogenesis, although its role in $\mathrm{SCl}$ development and progression remains controversial due to the inconsistent findings described. Summary: In this review, we summarize the current knowledge on the contribution of the NLRP3 inflammasome on potential neuroinflammation diseases therapy.

(c) 2020 S. Karger AG, Basel
\end{abstract}

\section{Introduction}

The immune system identifies and eradicates pathogens through the cooperation of the native (innate) immune system and the acquired immune system [1]. The native immune system acts as the initial line of defense that is implemented in the presence of cell-derived damage-associated molecular patterns (DAMPs) with or without the presence of infection [2]. These multifunctional molecules include heat shock proteins, messenger RNA, single-strand RNA, and small fragments of extracellular matrix that are released into the extracellular environment following tissue and cellular injury [3]. Furthermore, the natural immune system detects pathogenassociated molecular patterns (PAMPs) [2] derived from pathogens via pattern recognition receptor expressed by the cells of the innate immune system that recognize the microorganisms at the site of infection and present the antigens to the acquired immune system $[4,5]$.

In 2002, the first pattern recognition receptor (inflammasome) was discovered [6-8] following which, various inflammasomes have been identified comprising nodlike receptor protein (NLRP) 1, NLRiP2, NLRP3, absent in melanoma 2, and NLRC4 [9]. Among them, NLRP3 inflammasome is the best described as pyrin domain containing protein 3 [10]. NLRP3 is one of the most charac- 
terized of the inflammasomes that belongs to the NLRP family and contains 22 members in the human [11, 12]. NLRP3 reacts to a wide range of inflammatory infectious and endogenous ligands such as PAMPs and/or DAMPs; therefore, the dysregulation in the function of NLRP3 is associated with the pathogenesis of several inflammatory diseases $[13,14]$. This protein complex consists of $3 \mathrm{com}$ ponents including (a) NLRP3 scaffold, (b) PYCARD (PYD And CARD Domain) adapter, frequently referred to as apoptosis-associated speck-like protein (ASC), which functions as a caspase- 1 activator and (c) the third component is caspase- 1 . The NLRP3 complex is mainly expressed in immune cells, notably antigen-presenting cells and inflammatory cells after the inflammatory stimulatory trigger, which comprises macrophages (a potent antigen-presenting cell), dendritic cells, neutrophils in the spleen, and monocytes [15]. The 2 hit hypotheses have been proposed for NLRP3 activation [9]. The initial hit is when TLR is autophosphorylated by exposure to PAMPs and/or DAMPs and resulting in nuclear factor- $\kappa \mathrm{B}$ (NF$\kappa B)$ activation. This nuclear factor stimulates transcription and the expression of NLRP3 inflammasome components, proIL-1 $\beta$, and proIL-18, which after translocation from a nuclear to cytoplasmic location remain inactive until the second hit. This hypothesis is frequently evaluated in vitro via LPS [16]. The second hit assists the oligomerization of the inactive inflammasome complex (NLRP3, ASC, and caspase-1), which contributes to activation, maturation, and upregulation of interleukin (IL) $-1 \beta$ and IL-18 [17-19].

\section{Mechanisms of NLRP3-Activated Factors}

\section{The Role of ROS in the NLRP3 Inflammasomes}

Mitochondria and mitochondrial reactive oxygen species (mROS) is confirmed to induce the generation and maturation of IL-1 $\beta$ [20]. The clinical cohort study of patients with acute spinal cord injury showed that compared with the healthy control group, the blood or cerebrospinal fluid samples of these patients showed an increase in the expression of inflammatory proteins such as IL- $1 \beta$, as well as generation of ROS [21-25]. This study confirmed some pathological evidences related to spinal cord injury that ROS may induce inflammatory response. Recent researches have demonstrated that SCI induces the generation of ROS and the expression of NLRP3, ASC, pro-caspase-1, and proIL-1 $\beta$ [26-28]. When methylene blue, an antioxidant, was applied to spinal cord injury, these effects were significantly reduced. The results showed that the production of ROS was prior to the expression of ASC, NLRP3, caspase-1, and IL-1 $\beta$, which further supported ROS as one of the leading factors of NLRP3 initiation [26]. It is worth noting that, as noted above, ROS has been the result of interactions between trauma [23], ischemia-reperfusion [29], hypoxia [30], and other conditions [31, 32] of NLRP3 induced by a common mechanism, suggesting that ROS may be the major component required to trigger NLRP3 inflammasomes.

\section{The Role of Hypoxia in NLRP3 Inflammasomes}

Hypoxia is one of the important factors known to promote the production of inflammatory factors and participates in the pathogenesis of many diseases, especially in the central nervous system, which has been widely studied. Samples of SCI patients show increased production of IL-1 $\beta$. It has been pointed out that hypoxia can enhance the expression level of NLRP3 gene in microglia, thus promoting the production of IL- $1 \beta[33,34]$. In another study, animals with spinal cord ischemia/reperfusion injury, known as hypoxia induction, showed significant upregulation of NLRP3 and ASC expression compared to sham mice. In addition, the expression of NLRP3 RNA and protein level also increased significantly in the hypoxic-ischemic model of mouse central nervous system [29]. In addition, it is worth noting that hypoxia-inducible factor $1 \mathrm{~A}$ is an NF- $\kappa \mathrm{B}$-dependent transcription factor that reacts to hypoxia in cells or tissues. NLRP3 and PYCARD (ASC) genes are predicted to contain (hypoxiainducible factor 1A) binding sites, which means that hypoxia may be a key pathway to start inflammatory cells and may have significant synergistic effect with NF- $\kappa B$ activator in this regard $[21,35,36]$.

\section{The Role of Metabolites in NLRP3 Inflammasomes}

The concept that cellular metabolites can modulate the NLRP3 protein complex is newly proposed because of its altered content in some diseases associated with inflammatory activation. Although the view is still incomplete, some studies have shown that several metabolites can interfere with the inflammatory process. Dimethyl fumarate is a cell-permeable ester of the Krebs cycle metabolite dimethyl fumarate, which is thought to exert its beneficial effects mainly by reducing neuroinflammation, and also shows that in mouse innate immune cell lines. Among them, ROS production is reduced, most likely due to its upregulation of antioxidants such as heme oxygenase-1, superoxide dismutase-2, and nuclear factor erythroid 2 -related factor 2 , and reduction of IL- $1 \beta$ mRNA expression $[21,37,38]$, as shown by primary microglia. In addi- 
tion, the activity of the carbohydrate metabolism is also involved in the activation of NLRP3 inflammasomes [3941]. For example, 2-deoxy-D-glucose, a glycolysis inhibitor, has been shown to inhibit lipopolysaccharide-induced expression of proIL-1 $\beta$ and has recently been found to limit the production of reactive oxygen species to lipopolysaccharide and adenosine triphosphate, suggesting that its inhibition may be at least partially dependent [42]. It follows that the cascade of activation of inflammatory function requires the physiological process of energy metabolism to provide the necessary ATP, but whether the activity of the glycolytic pathway is controversial for the activation of NLRP3 inflammasomes [43].

\section{Spinal Cord Injury}

In SCI, primary injury of mechanical tissue leads to a variety of secondary injuries, including ion homeostasis [44], mitochondrial dysfunction [45], neurotransmitter release [46], and inflammatory response. Initiation of SCI secondary injury causes central nervous system innate immunity activation and can cause deleterious neurotoxic effects by releasing inflammatory cytokines such as IL$1 \beta$, TNF- $\alpha$, and ROS [47]. In the rats SCI model, activation of NLRP 3 inflammasomes occurs in the center of the lesion [21, 48, 49]. Moreover, our previous study found that treatment of mouse with zinc, a well-known ROS scavenger, was shown to reduce trauma-induced NLRP3 expression. Furthermore, treatment with zinc decreased the production of IL- $1 \beta$ and IL-18 via inhibiting inflammasome activation, but when zinc supplement was applied following monitoring ROS inhibitor, suggesting that zinc possibly originated from mitochondrial can block pyroptosis by intervening ROS level and downregulating NLRP3 inflammasomes complex expression [50].

\section{Neuroinflammation}

In the central nervous system, activation of NLRP3 inflammasomes is associated with amyloid and misfolded proteins [51]. In some pathological conditions, the misfolded protein itself cannot only act as a second messenger to promote the aggregation of NLRP3 protein complex, but also act as an initial signal molecule to initiate the transcription and expression of NLRP3 and IL-1 $\beta$, thus forming a positive feedback inflammatory cascade. However, this is not the only necessary way because the formation of large amounts of amyloid or misfolded pro- teins in the brain or spinal cord usually requires long chronic aseptic inflammation and complex biological processes, which can usually start NLRP3 inflammasomes by releasing other inflammatory molecules. Here, we highlight some examples of these 2 different inflammasomes, in which one of the most profound in vivo effects of inflammation initiation either directly or indirectly related to loss of NLRP3 related to amyloid and misfolded proteins is due to Alzheimer's disease. In AD mice model, the family form of mutant amyloid precursor protein and presenilin-1 is overexpressed in mice [12], and NLRP3 gene deletion has been shown to significantly improve object and spatial memory [13]. In vitro experiments also confirmed that amyloid protein cannot only act as a signal to activate inflammasomes but also bind to TLR4, trigger NF- $\mathrm{BB}$, and activate inflammasomes [16]. It is reported that familial amyotrophic lateral sclerosis is caused by the NLRP3 inflammasomes induced by another misfolded protein, mutant superoxide dismutase 1 . As a cytoplasmic protein, misfolded superoxide dismutase 1 is secreted out of the cell, and then triggers the TLR receptor of peripheral cells [12]. What is more, misfolded proteins may also act by acting on the endoplasmic reticulum in the cell, which may trigger NLRP3 activation signals through damaged mitochondria and ROS, such as HMGB1 in chronic diseases such as Parkinson's disease [8]. In addition, there may be attractive candidates to participate in the important process of inflammation initiation in other chronic neurodegenerative diseases. Even though aging has become a hot spot now, many of these findings still need to be verified in this field to seek to explain the differences mentioned.

\section{Conclusion}

Many pathways of inflammasomes play an important role in the aseptic inflammation of the host to the central nervous system, but the imbalance of NLRP3 inflammasomes is different from the process of necrosis and apoptosis, which is closely related to aging and trauma. With the clear understanding of the initiation, formation, activation, and secretion of inflammasomes, many promising inhibitors of inflammasomes have been developed, and the list of related anti-inflammatory drugs has been continuously improved. The steps of activation of inflammasomes may deepen our understanding of the pathogenicity of inflammasomes in aseptic inflammatory diseases of the nervous system. Research using neuroinflammation diseases and neurodegenerative diseases in knockout 
animal models suggested a protective role of inflammasome components against inflammation. Conversely, CNS trauma demonstrated that NLRP3 inflammasomes, IL-1 $\beta$ and IL-18, promote inflammation growth, proliferation, invasion, and metastasis. Furthermore, the evidence suggesting that the NLRP3 inflammasome is contributing to neuroinflammation diseases progression in vivo remains preliminary and requires further confirmation. It has been suggested that inflammasome activation in CNS specifically depend on the tissue context to whether inhibition or activation of results. Further studies are required to address the molecular mechanisms behind the production, activation, and modulation of inflammasomes and to determine their potential therapeutic role in human CNS diseases. In addition, in this more and more in-depth field, this information may reveal a clear opportunity to develop new therapeutic interventions to treat such diseases in clinic.

\section{Acknowledgement}

This work was supported by the National Natural Science Foundation of China (No. 81671907 and 81871556).

\section{Conflict of Interest Statement}

The authors have declared that they have no conflict of interests and ethics for this work and project.

\section{Funding Sources}

This work was supported by the National Natural Science Foundation of China (Nos. 81671907 and 81871556).

\section{Author Contributions}

Sen Lin contributed in study design and manuscript preparation. Xifan Mei contribution in fund collection.

\section{References}

1 Neill DR, Wong SH, Bellosi A, Flynn RJ, Daly $\mathrm{M}$, Langford TK, et al. Nuocytes represent a new innate effector leukocyte that mediates type-2 immunity. Nature. 2010;464(7293): 1367-70.

2 Abderrazak A, Syrovets T, Couchie D, El Hadri K, Friguet B, Simmet T, et al. NLRP3 inflammasome: from a danger signal sensor to a regulatory node of oxidative stress and inflammatory diseases. Redox Biol. 2015;4: 296-307.

3 Strowig T, Henao-Mejia J, Elinav E, Flavell R. Inflammasomes in health and disease. Nature. 2012;481(7381):278-86.

4 Dolasia K, Bisht MK, Pradhan G, Udgata A, Mukhopadhyay S. TLRs/NLRs: shaping the landscape of host immunity. Int Rev Immunol. 2018;37(1):3-19.

5 Hamon MA, Quintin J. Innate immune memory in mammals. Semin Immunol. 2016; 28(4):351-8.

6 Sanders MG, Parsons MJ, Howard AG, Liu J, Fassio SR, Martinez JA, et al. Single-cell imaging of inflammatory caspase dimerization reveals differential recruitment to inflammasomes. Cell Death Dis. 2015;6:e1813.

7 Gentile LF, Cuenca AL, Cuenca AG, Nacionales DC, Ungaro R, Efron PA, et al. Improved emergency myelopoiesis and survival in neonatal sepsis by caspase-1/11 ablation. Immunology. 2015;145(2):300-11.

8 Jorgensen I, Miao EA. Pyroptotic cell death defends against intracellular pathogens. Immunol Rev. 2015;265(1):130-42.

9 Saïd-Sadier N, Ojcius DM. Alarmins, inflammasomes and immunity. Biomed J. 2012; 35(6):437-49.
10 Rathinam VA, Fitzgerald KA. Inflammasome complexes: emerging mechanisms and effector functions. Cell. 2016;165(4):792-800.

11 Sharma D, Kanneganti TD. The cell biology of inflammasomes: mechanisms of inflammasome activation and regulation. J Cell Biol. 2016;213(6):617-29.

12 Lang Y, Chu F, Shen D, Zhang W, Zheng C, Zhu J, et al. Role of inflammasomes in neuroimmune and neurodegenerative diseases: a systematic review. Mediators Inflamm. 2018; 2018:1549549.

13 Labzin LI, Heneka MT, Latz E. Innate immunity and neurodegeneration. Annu Rev Med. 2018;69:437-49.

14 Mortezaee K, Khanlarkhani N, Beyer C, Zendedel A. Inflammasome: its role in traumatic brain and spinal cord injury. J Cell Physiol. 2018;233:5160-9.

15 Shao BZ, Xu ZQ, Han BZ, Su DF, Liu C. NLRP3 inflammasome and its inhibitors: a review. Front Pharmacol. 2015;6:262.

16 Franchi L, Eigenbrod T, Muñoz-Planillo R, Ozkurede U, Kim YG, Arindam C, et al. Cytosolic double-stranded RNA activates the NLRP3 inflammasome via MAVS-induced membrane permeabilization and $\mathrm{K}+$ efflux. J Immunol. 2014;193(8):4214-22.

17 Song N, Li T. Regulation of NLRP3 inflammasome by phosphorylation. Front Immunol. 2018;9:2305.

18 Groslambert M, Py BF. Spotlight on the NLRP3 inflammasome pathway. J Inflamm Res. 2018;11:359-74.

19 Latz E, Duewell P. NLRP3 inflammasome activation in inflammaging. Semin Immunol. 2018;40:61-73.
20 Sandhir R, Halder A, Sunkaria A. Mitochondria as a centrally positioned hub in the innate immune response. Biochim Biophys Acta. 2017;1863(5):1090-7.

21 Ravindran R, Loebbermann J, Nakaya HI, Khan N, Ma H, Gama L, et al. The amino acid sensor GCN2 controls gut inflammation by inhibiting inflammasome activation. Nature. 2016;531(7595):523-7.

22 Albayar AA, Roche A, Swiatkowski P, Antar S, Ouda N, Emara E, et al. Biomarkers in spinal cord injury: prognostic insights and future potentials. Front Neurol. 2019;10:27.

23 Batchelor PE, Tan S, Wills TE, Porritt MJ, Howells DW. Comparison of inflammation in the brain and spinal cord following mechanical injury. J Neurotrauma. 2008;25(10): 1217-25.

24 Tang Y, Liu HL, Min LX, Yuan HS, Guo L, Han PB, et al. Serum and cerebrospinal fluid tau protein level as biomarkers for evaluating acute spinal cord injury severity and motor function outcome. Neural Regen Res. 2019; 14(5):896-902.

25 Sarkis GA, Mangaonkar MD, Moghieb A, Lelling B, Guertin M, Yadikar H, et al. The application of proteomics to traumatic brain and spinal cord injuries. Curr Neurol Neurosci Rep. 2017;17(3):23.

26 Lin ZH, Wang SY, Chen LL, Zhuang JY, Ke QF, Xiao DR, et al. Methylene blue mitigates acute neuroinflammation after spinal cord injury through inhibiting NLRP3 inflammasome activation in microglia. Front Cell Neurosci. 2017;11:391.

27 Hennig P, Garstkiewicz M, Grossi S, Di Filippo M, French LE, Beer HD. The crosstalk between Nrf2 and inflammasomes. Int J Mol Sci. 2018; $19: 562$. 
28 Jiang W, Li M, He F, Yao W, Bian Z, Wang X, et al. Protective effects of asiatic acid against spinal cord injury-induced acute lung injury in rats. Inflammation. 2016;39(6):1853-61.

29 Yao W, Tai LW, Liu Y, Hei Z, Li H. Oxidative stress and inflammation interaction in ischemia reperfusion injury: role of programmed cell death. Oxid Med Cell Longev. 2019;2019: 6780816.

30 Qiu Z, He Y, Ming H, Lei S, Leng Y, Xia ZY. Lipopolysaccharide (LPS) aggravates high glucose- and hypoxia/reoxygenation-induced injury through activating ROS-dependent NLRP3 inflammasome-mediated pyroptosis in H9C2 cardiomyocytes. J Diabetes Res. 2019;2019:8151836.

31 Chen X, Guo X, Ge Q, Zhao Y, Mu H, Zhang J. ER stress activates the NLRP3 inflammasome: a novel mechanism of atherosclerosis. Oxid Med Cell Longev. 2019;2019:3462530.

32 Wei J, Wang $\mathrm{H}$, Wang $\mathrm{H}$, Wang B, Meng L, Xin Y, et al. The role of NLRP3 inflammasome activation in radiation damage. Biomed Pharmacother. 2019;118:109217.

33 Ystgaard M, Scheffler K, Suganthan R, Bjørås M, Ranheim T, Sagen E, et al. Neuromodulatory effect of NLRP3 and ASC in neonatal hypoxic ischemic encephalopathy. Neonatology. 2019;115(4):355-62.

34 Ding HG, Deng YY, Yang RQ, Wang QS, Jiang WQ, Han YL, et al. Hypercapnia induces IL- $1 \beta$ overproduction via activation of NLRP3 inflammasome: implication in cognitive impairment in hypoxemic adult rats. J Neuroinflammation. 2018;15(1):4.

35 Martínez-García JJ, Martínez-Banaclocha $\mathrm{H}$, Angosto-Bazarra D, de Torre-Minguela C, Baroja-Mazo A, Alarcón-Vila C, et al. P2X7 receptor induces mitochondrial failure in monocytes and compromises NLRP3 inflammasome activation during sepsis. Nat Commun. 2019;10(1):2711.

36 Mian Wu M, Zhang M, Ma Y, Liu F, Chen S, $\mathrm{Lu} \mathrm{J}$, et al. Chaetocin attenuates gout in mice through inhibiting HIF-1 $\alpha$ and NLRP3 inflammasome-dependent IL-1 $\beta$ secretion in macrophages. Arch Biochem Biophys. 2019; 670:94-103.

37 Peng H, Li H, Sheehy A, Cullen P, Allaire N, Scannevin RH. Dimethyl fumarate alters microglia phenotype and protects neurons against proinflammatory toxic microenvironments. J Neuroimmunol. 2016;299:35-44.

38 Paraiso HC, Kuo PC, Curfman ET, Moon HJ, Sweazey RD, Yen JH, et al. Dimethyl fumarate attenuates reactive microglia and long-term memory deficits following systemic immune challenge. J Neuroinflammation. 2018;15(1): 100.

39 Próchnicki T, Latz E. Inflammasomes on the crossroads of innate immune recognition and metabolic control. Cell Metab. 2017;26(1): 71-93.

40 McGettrick AF, O'Neill LA. NLRP3 and IL-1 $\beta$ in macrophages as critical regulators of metabolic diseases. Diabetes Obes Metab. 2013; 15(Suppl 3):19-25.

41 Tannahill GM, O'Neill LA. The emerging role of metabolic regulation in the functioning of toll-like receptors and the NOD-like receptor Nlrp3. FEBS Lett. 2011;585(11):1568-72.

42 Zhong WJ, Yang HH, Guan XX, Xiong JB, Sun CC, Zhang CY, et al. Inhibition of glycolysis alleviates lipopolysaccharide-induced acute lung injury in a mouse model. J Cell Physiol. 2019;234(4):4641-54.
43 Nomura J, So A, Tamura M, Busso N. Intracellular ATP decrease mediates NLRP3 inflammasome activation upon nigericin and crystal stimulation. J Immunol. 2015;195(12):5718-24.

44 Liu WM, Wu JY, Li FC, Chen QX. Ion channel blockers and spinal cord injury. J Neurosci Res. 2011;89:791-801.

45 Scholpa NE, Schnellmann RG. Mitochondrial-based therapeutics for the treatment of spinal cord injury: mitochondrial biogenesis as a potential pharmacological target. J Pharmacol Exp Ther. 2017;363(3):303-13.

46 Keefe KM, Sheikh IS, Smith GM. Targeting neurotrophins to specific populations of neurons: NGF, BDNF, and NT-3 and their relevance for treatment of spinal cord injury. Int J Mol Sci. 2017;18(3):548.

47 Wang JF, Li Y, Song JN, Pang HG. Role of hydrogen sulfide in secondary neuronal injury. Neurochem Int. 2014;64:37-47.

48 Yamamoto S, Yamashina K, Ishikawa $\mathrm{M}$, Gotoh M, Yagishita S, Iwasa K, et al. Protective and therapeutic role of 2-carba-cyclic phosphatidic acid in demyelinating disease. J Neuroinflammation. 2017;14(1): 142.

49 Jiang W, Li M, He F, Zhou S, Zhu L. Targeting the NLRP3 inflammasome to attenuate spinal cord injury in mice. J Neuroinflammation. 2017;14(1):207.

50 Katsumoto A, Takeuchi H, Takahashi K, Tanaka F. Microglia in Alzheimer's disease: risk factors and inflammation. Front Neurol. 2018;9:978.

51 Heneka MT, McManus RM, Latz E. Inflammasome signalling in brain function and neurodegenerative disease. Nat Rev Neurosci. 2018;19(10):610-21. 